\title{
Design of optimal control systems with bounded control signals
}

\author{
Wolfgang Reinelt \\ Division of Automatic Control \\ Department of Electrical Engineering \\ Linköpings universitet, SE-581 83 Linköping, Sweden \\ WWW: http://WwW. control.isy.liu.se \\ Email: wolle@isy.liu.se
}

May 2001

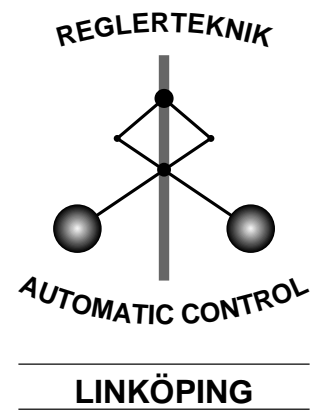

Report No.: LiTH-ISY-R-2350

Submitted to ECC 2001, Porto, Portugal

Technical reports from the Automatic Control group in Linköping are available by anonymous ftp at the address ftp.control.isy.liu.se. This report is contained in the file 2350.pdf. 


\title{
DESIGN OF OPTIMAL CONTROL SYSTEMS WITH BOUNDED CONTROL SIGNALS
}

\author{
Wolfgang Reinelt \\ Dept of Electrical Eng., Linköping University, 58183 Linköping, Sweden. \\ E-mail: wolle@isy.liu.se, http://www.control.isy.liu.se/wolle/
}

Keywords: Constraint Control, Saturation Avoidance, Hard Bounds, Optimal Control Systems, Youla Parameterisation.

\begin{abstract}
The design of optimal controllers for systems subject to hard bounds on the control signal is considered. Optimality refers to the fact that we achieve the smallest possible worst case error (i.e. difference between reference signal and plant output) during runtime. The external reference signal is bounded in amplitude and rate, which encounters for many practical situations. Moreover, a conservative design is avoided by these assumptions. The resulting constrained and infinite dimensional optimisation problem is re-casted to an unconstrained and finite dimensional one by applying the notion of Pareto-optimal solutions and Ritz approximation. Moreover, the presented framework allows to assess feasibility of the constraint control problem, and to display the tradeoff between the two objectives. A simulation example illustrates the developed theory.
\end{abstract}

\section{Introduction and Motivation}

Most practical control problems are dominated by hard bounds. Valves can only be operated between fully open and fully closed, pumps and compressors have a finite throughput capacity and tanks can only hold a certain volume. These inputor actuator-bounds convert the linear model into a nonlinear one. Exceeding these prescribed bounds causes unexpected behaviour of the system - large overshoots, low performance or (in the worst case) instability. A classical example for the detrimental effect of neglecting constraints is the Chernobyl nuclear power plant disaster in 1986. Solving control problems subject to hard bounds in a linear framework, we necessarily need to restrict the amplitudes of the external signals (i.e. the reference signals) as well. In this approach, we look at reference signals, bounded in amplitude and rate (i.e. the first derivative has to be bounded in its amplitude). This appears in many systems. For example in a tank, not only the liquid-level is bounded (by the tanks height), additionally the liquid cannot change its level arbitrarily fast.

In this work we consider the design of optimal controllers, guaranteeing prescribed hard bounds on the control signal. By optimality we mean, that the designed controller is the one guaranteeing the smallest error during runtime, where error is defined as the amplitude of the difference between reference signal and plant output. The definition of this error by a hard bound fits into the framework of hard bounded control and reference signals. Moreover, the presented framework allows us to assess feasibility of the constraint control problem.

Design of controllers for systems with hard constraints is a quite vivid area of research, see for example the recent books $[10,19,23]$ or special issues $[2,21]$ and the references therein. The problem has been addressed within the Model Predictive Control community [11] and another popular approach is to use so-called Anti Windup Bumpless Transfer schemes [1, 12].

Controller designs that encounter the saturation effect a-priori are usually separated into two categories: (1) designs that prevent saturation of the control signal and therefore enjoy a linear framework (as long as plant and controller are linear) and (2) methods that allow saturation and are therefore facing a nonlinear setup. In the second case, analysis (in terms of stability, controllability and feasibility) of this nonlinear system is discussed in $[6,20,22]$. Design schemes that handle saturations using a (nonlinear) control law have recently been proposed in $[18,5]$ for instance.

This work clearly employs the first - saturation avoiding - philosophy. To solve the constraint control problem in a linear framework, one implicitly has to restrict the amplitude of all external signals; independent of the technique used in particular. Our approach, however, makes a further step by imposing an additional restriction on the rate of the external signals. In many practical situations, this is a more accurate description (than without rate restriction) of all external signals, possibly arising during runtime. A design, directly based on this description will avoid a conservative control system.

Using Youla parameterisation, our approach is somewhat related to [8]; the focus there, however is more on the interplay between stability and initial conditions, which we do not discuss here. On the other hand, external (reference) signals are not present and optimality is not treated in [8], which is our main emphasis in this work.

Based on the same description of external signals, related works studied energy bounds instead of hard bounds [9] or allow some process noise [14]. Uncertain or multivariable systems with respect to hard constraints have been studied in $[16,17]$.

Paper outline: Sec. 2 poses the problem and repeats some standard results on Youla parameterisation for reading convenience and reference purposes. Our main technical contribution is given in Sec. 3, where the infinite dimensional optimi- 
sation problem is re-casted to a finite dimensional and unconstrained one. The remaining technical detail, the calculation of maximum control amplitude and maximum error, was already examined in recent works [15], and is stated shortly in Sec. 4. An illustrative example is given in Sec. 5. We summarise the work in Sec. 6.

\section{Notation and Problem Formulation}

We study control systems with reference signals, bounded in amplitude and rate. In this work, we restrict ourselves to linear, time-invariant, single-input single output plants, represented by a transfer function $G$. The goal is to design a linear, time invariant controller such that a prescribed hard bound of the control signal is not exceeded for a certain set of external signals (cf. Def. 1 and 2 below). For this standard setup, see also Fig. 1,

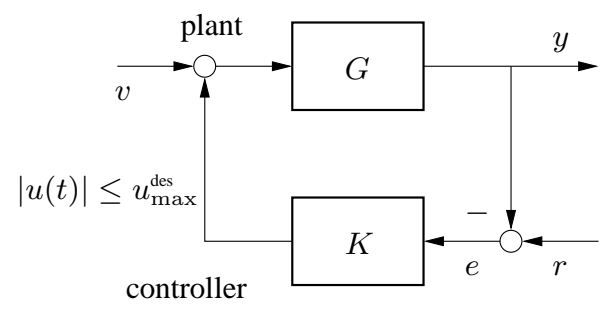

Figure 1: Control system with output $y$, constraint control variable $u$, reference signal $r$, error $e$ and process noise $v$. $u_{\max }^{d e s}$ denotes the desired upper bound for the control signal.

we have the following relation between external signals $r$ and $v$ and internal signals $e$ and $u$, respectively:

$$
\begin{aligned}
\left(\begin{array}{c}
e \\
u
\end{array}\right) & =H(G, K) \cdot\left(\begin{array}{c}
r \\
v
\end{array}\right) \\
H(G, K) & =\left(\begin{array}{cc}
(I+G K)^{-1} & -(I+G K)^{-1} G \\
K(I+G K)^{-1} & I-K(I+G K)^{-1} G
\end{array}\right) .
\end{aligned}
$$

It it well-known, that all stabilising controllers can be parameterised in a very convenient and elegant way by the so-called Youla parameterisation, see [25, ch.5.2] for details. Therefore, we assume a coprime factorisation of the plant $G=\bar{D}_{G}^{-1} \bar{N}_{G}=$ $N_{G} D_{G}^{-1}$ and a stabilising controller $K_{s}=N_{K} D_{K}^{-1}$, where the coprime factors of the controller obey the Bezout equation $\bar{D}_{G} D_{K}+\bar{N}_{G} N_{K}=1$, which is a necessary and sufficient condition for stabilisation of the plant $G$. The stabilising controller $K_{s}$ can be realized as a full state feedback and observer, cf. [7, App.A.4]. Then, all stabilising controllers are given by

$$
K=K(Q)=\left(N_{K}+D_{G} Q\right)\left(D_{K}-N_{G} Q\right)^{-1}
$$

where $Q$ is any stable transfer function (short: $Q \in \mathcal{H}_{\infty}$ ). The beauty of this approach is that system (1) is now affine in the so-called Youla parameter $Q$ :

$H(G, K)=\left(\begin{array}{cc}\left(D_{K}-N_{G} Q\right) \bar{D}_{G} & -\left(D_{K}-N_{G} Q\right) \bar{N}_{G} \\ \left(N_{K}+D_{G} Q\right) \bar{D}_{G} & I-\left(N_{K}+D_{G} Q\right) \bar{N}_{G}\end{array}\right)$

and one can vary $Q$ until desired properties of the system (3) are obtained.
Having established the standard notation on the control system, we now turn to the definitions for admissible reference signal and maximum control signal, which are quite straightforward from the motivation:

Definition 1 (Admissible Reference Signal) Given real values $R, \dot{R}>0$, then a reference signal $r$ is called $(R, \dot{R})$ admissible, if the following properties hold:

1. $|r(t)| \leq R$ for all $t>0$ and

2. $|\dot{r}(t)| \leq \dot{R}$ for all $t>0$.

The set of all $(R, \dot{R})$-admissible reference signals is denoted by $\mathcal{A}(R, \dot{R})$.

Definition 2 (Maximum Control Signal) Assume the control system in Fig. 1 to be internally stable (that is, the expression in (1) is proper and stable [7, App.A]). We call

$$
u_{\max }:=\sup \left\{\|u\|_{\infty} ; \forall r \in \mathcal{A}(R, \dot{R})\right\}
$$

the Maximum Control Signal.

It clearly depends not only on the reference signals but on plant and controller as well and its calculation is discussed in Sec. 4. Based on this formal description of the control system, we state the following:

Definition 3 (Optimality of the Constraint Control System) The internally stable control system made up with plant $G$ and controller $K$ as in Fig. 1, is called optimal, if and only if the following properties for all $(R, \dot{R})$-admissible reference signals hold:

1. Saturation Avoidance: The control signal must not exceed the prescribed or desired bound $u_{\max }^{\text {des }}$ for all times:

$$
u_{\max } \leq u_{\max }^{\text {des }}
$$

2. Optimality: the maximum error, i.e. the difference between reference signal and plant output, should be minimal in the following sense:

$$
e_{\text {max }}:=\sup \left\{\|r-y\|_{\infty} ; \forall r \in \mathcal{A}(R, \dot{R})\right\} \rightarrow \min .
$$

Remark 1 To demand a rate restriction on the reference signal makes the minimisation in (6) nontrivial. Consider for example the system as in Fig. 1 with a transfer function from reference signal $r$ to error signal e (degree of numerator equal to degree of denominator), so that a step as reference signal will invoke $e(0)=1$. Then no minimisation will reduce the maximum error to a value $e_{\max }<1$. In this sense, property (2.) in Def. 1 is crucial for the statement of the optimisation problem. 
Remark 2 The maximum control amplitude $u_{\max }$ as defined in (4) as well as the maximum error $e_{\max }$ in (6) depend both on the set of admissible reference signals. Assuming now an iterative minimisation of the maximum error, we need to calculate the maximum control amplitude $u_{\max }$ as well as the maximum error $e_{\max }$ in each iteration step. The problem, how to calculate the maximum output of a system, where the input is bounded in amplitude and rate has already been addressed in [14, 15] and is stated in Sec. 4.

The infinite dimensional and constrained optimisation problem, hidden in Def. 3, will be reduced to a finite dimensional and unconstrained one in the following section.

\section{The Optimisation Problem}

Using the Youla parameterisation of the control system, the design of the optimal controller can be stated as follows:

Optimisation Problem 1 By variation of the Youla parameter in (3), minimise the error $e_{\max }=e_{\max }(Q)$ in (6), so that the maximum control signal $u_{\max }=u_{\max }(Q)$ does not exceed the bound $u_{\max }^{\text {des }}$ prescribed in (5), i.e.:

$$
\begin{aligned}
Q^{*}= & \arg \min _{Q \in \mathcal{H}_{\infty}} e_{\max }(Q) \\
& \text { such that } u_{\max }(Q) \leq u_{\max }^{\text {des }}
\end{aligned}
$$

This is an infinite dimensional optimisation problem, because the parameter space (i.e. all admissible Youla parameters $Q$ ) is infinite dimensional. Moreover, the optimisation objective (7) is constraint by (8). First, we will eliminate the constraint. Therefore, we state a standard result from convex optimisation [4]:

Proposition 1 Let $X$ be a convex set and $f, g: X \rightarrow \mathbb{R}^{+}$ convex functionals. Then the following are equivalent:

(a) A so-called Pareto optimal point $x^{*} \in X$ exists. I.e. there is no $x \in X \backslash\left\{x^{*}\right\}$ such that $f(x) \leq f\left(x^{*}\right), g(x) \leq g\left(x^{*}\right)$ hold with at least one of the two inequalities being strict.

(b) There exists an $\alpha \in[0 ; 1]$ such that $x^{*}$ minimises $\alpha f(x)+$ $(1-\alpha) g(x)$ over $X$.

The definition of Pareto-optimal points is obviously useful to eliminate suboptimal solutions in a joint minimisation of $f$ and $g$ over $X$. In turn, this equivalence characterises the set of all Pareto-optimal points of $f, g$ via a minimisation of their convex combination, which is an unconstrained minimisation. We will apply Proposition 1 to our problem, see [9, 24] for discussions in the same spirit.

Both, $e_{\max }$ and $u_{\max }$, are convex functionals in the Youla parameter $Q$ (to see this, note that all entries of $H$ in (3) are affine in $Q$, then apply triangle inequality). Moreover, both functionals are obviously non-negative and the set of Youla parameters (all stable transfer functions $\mathcal{H}_{\infty}$ ) is a convex set. Consider the minimisation of the following family of convex optimisation problems:

$Q^{*}(\alpha):=\arg \min _{Q \in \mathcal{H}_{\infty}} \alpha \cdot e_{\max }(Q)+(1-\alpha) u_{\max }(Q), \quad \alpha \in[0 ; 1]$.

which will yield the set of Pareto-optimal Youla parameters $Q^{*}(\alpha)$. We can state the following property of $e_{\max }\left(Q^{*}(\alpha)\right)$ and $u_{\max }\left(Q^{*}(\alpha)\right)$ as functions of $\alpha$ :

Proposition $2 e_{\max }\left(Q^{*}(\alpha)\right)$ is a monotone decreasing and $u_{\max }\left(Q^{*}(\alpha)\right)$ a monotone increasing function in $\alpha \in[0 ; 1]$.

Proof. Assume the contrary: $\alpha_{1}<\alpha_{2}$ and $e_{\max }\left(Q^{*}\left(\alpha_{1}\right)\right)<$ $e_{\max }\left(Q^{*}\left(\alpha_{2}\right)\right)$. The optimality of $Q^{*}\left(\alpha_{k}\right)$, cf. Proposition 1 and $(9)$, yields:

$$
\begin{aligned}
& \alpha_{1}\left(e_{\max }\left(Q^{*}\left(\alpha_{2}\right)\right)-e_{\max }\left(Q^{*}\left(\alpha_{1}\right)\right)\right)+ \\
& \left(1-\alpha_{1}\right)\left(u_{\max }\left(Q^{*}\left(\alpha_{2}\right)\right)-u_{\max }\left(Q^{*}\left(\alpha_{1}\right)\right)\right) \geq 0 \\
& \alpha_{2}\left(e_{\max }\left(Q^{*}\left(\alpha_{1}\right)\right)-e_{\max }\left(Q^{*}\left(\alpha_{2}\right)\right)\right)+ \\
& \left(1-\alpha_{2}\right)\left(u_{\max }\left(Q^{*}\left(\alpha_{1}\right)\right)-u_{\max }\left(Q^{*}\left(\alpha_{2}\right)\right)\right) \geq 0 .
\end{aligned}
$$

Combining these two inequalities yields

$$
\begin{aligned}
\left(\frac{\alpha_{2}}{1-\alpha_{2}}-\frac{\alpha_{1}}{1-\alpha_{1}}\right) \underbrace{\left(e _ { \operatorname { m a x } } \left(Q^{*}\left(\alpha_{1}\right)-e_{\max }\left(Q^{*}\left(\alpha_{2}\right)\right)\right.\right.}_{<0, \text { by assumption }} & \geq 0 \\
\Leftrightarrow \frac{\alpha_{2}}{1-\alpha_{2}}-\frac{\alpha_{1}}{1-\alpha_{1}} & \leq 0 \\
\Leftrightarrow \alpha_{1} & \geq \alpha_{2}
\end{aligned}
$$

which contradicts our assumption. The proof for $u_{\max }\left(Q^{*}(\alpha)\right)$ is similar.

Having calculated the Pareto-optimal Youla parameters, we obtain two monotone functions $u_{\max }\left(Q^{*}(\alpha)\right), e_{\max }\left(Q^{*}(\alpha)\right)$ in the parameter $\alpha$, see Fig. 2. We are interested in the Youla parameter,that guarantees the on the maximum control amplitude $u_{\max } \leq u_{\max }^{\text {des }}$, which draws our attention to the interval $\left[0, \alpha^{*}\right]$ in which $u_{\max }\left(Q^{*}(\alpha)\right) \leq u_{\max }^{\text {des }}$ holds and it is clear, that $Q=Q^{*}\left(\alpha^{*}\right)$ is the optimal solution to our constrained problem, as it obeys the bound on the control amplitude with the smallest error.

Proposition 2 is highly intuitive, as a smaller error should only be achieved applying a larger (at least with no smaller) control signal. Note, however, that the minimisation as stated in (9), is independent of the prescribed bound for the control signal $u_{\max }^{\text {des }}$. The set of Pareto-optimal Youla-parameters might deliver a larger control signal as prescribed, which makes the constraint control problem as stated in Def. 3 infeasible. This allows us to state a quite simple feasibility criterion and to reformulate Optimisation Problem 1:

Proposition 3 (Feasibility) The constraint control problem 3 is feasible, if and only if there exists an $\alpha \in[0 ; 1]$ so that $u_{\max }\left(Q^{\alpha}\right) \leq u_{\max }^{\text {des }}$ holds. 


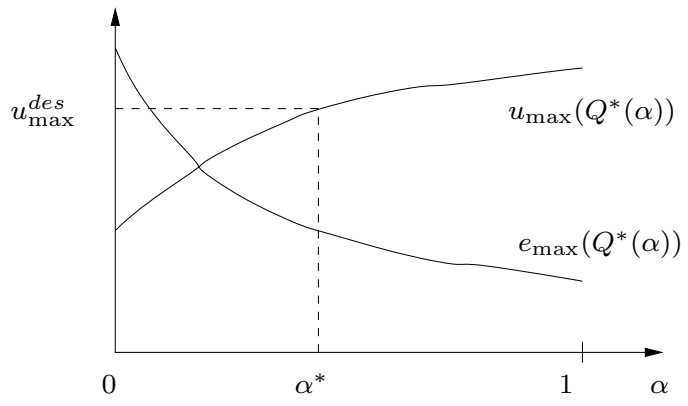

Figure 2: Maximum control amplitude $u_{\max }\left(Q^{\alpha}\right)$ and maximum error $e_{\max }\left(Q^{\alpha}\right)$.

Optimisation Problem 2 1. Calculate $Q^{*}(\alpha)$ for all $\alpha \in$ [0;1], see eqn. (9)

2. Determine $\alpha^{*} \in[0 ; 1]$ with $\max _{\alpha}\left\{u_{\max }\left(Q^{*}(\alpha)\right)\right\} \leq$ $u_{\max }^{\text {des }}$. If no such $\alpha^{*}$ exists, exit (problem infeasible).

3. Let $Q^{\text {opt }}=Q^{*}\left(\alpha^{*}\right)$ and calculate the optimal controller via (2).

In practice, however, the first step has to be performed on an $\alpha$-grid instead of the hole interval. The monotony of both $u_{\max }\left(Q^{\alpha}\right)$ and $e_{\max }\left(Q^{\alpha}\right)$ ensures, that nothing "unexpected" can happen between two grid points. Moreover, the grid can be refined using a simple binary search near the maximum control amplitude of interest.

The infinite dimension of the parameter space $Q \in \mathcal{H}_{\infty}$ is still present in the first step of the optimisation scheme, but will be removed in a standard way now using Ritz approximation [13]. As all Youla parameters are stable, we use the mapping $z:=\frac{s-p}{s+p}$ to map the closed right half plane into the closed unit circle. Now, $Q=Q(z)$ is analytic on the unit circle and $Q(z)=\sum_{\nu=1}^{\infty} X_{\nu} z^{\nu-1}$ will converge uniformly. Thus, we will approximate the Youla parameter $Q$ by the finite sum $\hat{Q}_{N}(s)=\sum_{\nu=1}^{N} X_{\nu}\left(\frac{s-p}{s+p}\right)^{\nu-1}$. As both $e_{\max }$ and $u_{\max }$ are continuous in $Q^{*}$ (because of the optimality of $Q^{*}$ ), we can use the approximated Youla parameter for approximation of error and maximum control signal. This approximation, however, is most welcome from the engineering point of view, as it limits the order of the controller. The actual computational task is to obtain the (now finite number of) coefficients $X_{\nu}$, which involves non-linear optimisation.

Remark 3 If we are only interested in computing the optimal constraint control system with a given bound $u_{\max }^{\text {des }}$, then one might consider solving problem $(7,8)$ instead of $(9)$ on the $\alpha$ grid, as both problems involve non-linear optimisation (due to the Ritz-approximation). However, problem $(7,8)$ is a constrained optimisation problem and thus harder to solve than the unconstrained problem (9). Moreover, it is easier to find an initial value for the unconstrained problem: considering first the grid point $\alpha=0$ with initial values $X_{\nu}=0$ and then in- crease $\alpha$, taking the resulting $X_{\nu}$ 's of the "old" grid point as initial value for the next one.

\section{Calculation of Maximum Control Amplitude and Maximum Error}

As mentioned in Remark 2, we are in need to compute maximum control amplitude and maximum error during the minimisation in step 1 of Optimisation Problem 2. In one minimisation step, the Youla parameter $Q$ is fixed and so the control system is known completely. Thus for the given set of admissible reference signals $\mathcal{A}(R, \dot{R})$, plant $G$ and controller given by $K(Q)$, we need to calculate maximum control amplitude and maximum error. This problem has been studied in $[14,15]$. We state the main result, that is interesting for this work, formally as:

\section{Theorem 1 (Calculation of the Maximum Output Signal)} Given a linear and time invariant SISO system $P$ with input signal $x$ and output signal $z$. Let the reference signal be $(X, \dot{X})$-admissible.

(a) There exists an algorithm that determines the maximum output amplitude $z_{\max }$ according to Def. 2 of this system for all admissible input signals.

(b) An $(X, \dot{X})$-admissible input exists, so that $z_{\max }$ is achieved.

The algorithm outlined in the original work [14] works for SISO systems and constructs the worst case input $x$ (mentioned under (b) above), a method, that turns out to be time consuming and complicated to implement. Therefore, an improved numerical solution based on Linear Programming techniques has been derived in [15], where the extension to multivariable systems is discussed as well. Uncertain systems can be handled as well [17].

Independent of the particular numerical solution, however, Theorem 1 can be used to determine the maximum control amplitude $u_{\text {max }}$, when the reference signal $r$ of the control system is $(R, \dot{R})$-admissible: let therefore $x=r, z=u$ and the system $P$ in Theorem 1 is $P=K(I+G K)^{-1}$, the transfer function from $r$ to $u$. In the same way, the maximum error $e_{\max }$ can be obtained: let $x=r, z=e$ and $P=(I+G K)^{-1}$, the transfer function from $r$ to $e$. Thus, applying Theorem 1 two times per minimisation step, we are able to solve Optimisation Problem 2 numerically.

\section{Illustrative Example}

We study the unstable and non-minimum phase plant $G(s)=$ $\frac{s-1}{s(s-2)}$ in the standard setup as depicted in Fig. 1. As shown in [25] are all controllers stabilising this plant, unstable and at least of order one. We assume the disturbance to be zero while the reference signal shall have the limitations: $|r(t)| \leq$ $1,|\dot{r}(t)| \leq 0.8$. As seen above, the design procedure enables a 

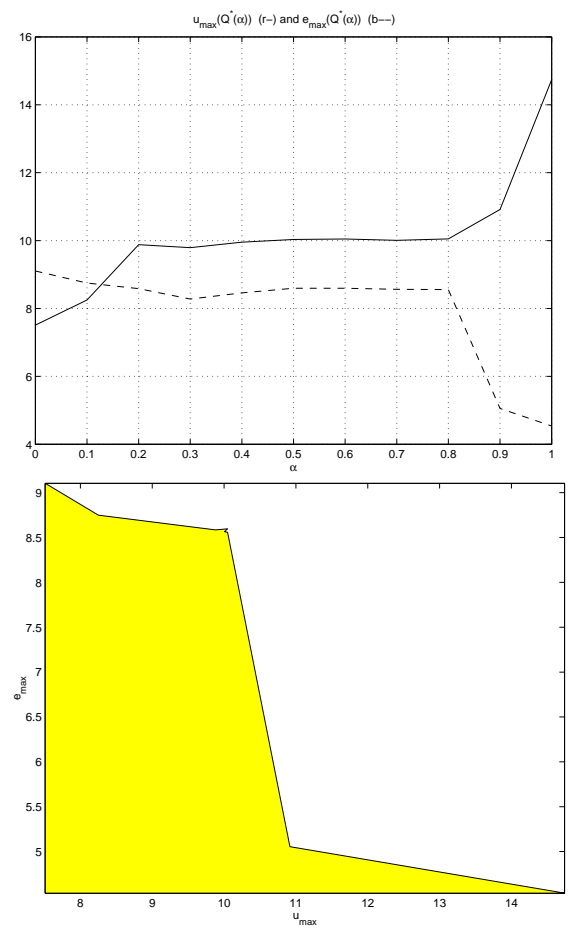

Figure 3: Maximum error $e_{\max }$ and control amplitude $u_{\max }$. Top: as monotone functions in $Q^{*}(\alpha)$, bottom: as tradeoffcurve over $\alpha$ in the $u_{\max } / e_{\max }$ plane. The shaded area indicates those combinations of maximum error and maximum control amplitude, that cannot be reached.

feasibility check of the constraint control problem, thus it is not necessary to specify the maximum of the control signal $u_{\max }^{\text {des }}$ at this stage.

In order to replace the infinite dimensional optimisation problem (as outlined in the end of Sec. 3) by a finite one, we approximate the Youla parameter choosing $p=2$ and $N=6$ in the Ritz approximation. The parameter $N$ prescribes the order of the Youla parameter and thus (implicitly) the order of the controller. To remove the constraint $u_{\max } \leq u_{\max }^{\text {des }}$ in the optimisation problem, we solve the convex family of optimisation problems in (9) on a equidistant grid on $0 \leq \alpha \leq 1$ (ten grid points) instead.

Moreover, Proposition 2 stated the monotony of the functions $e_{\max }$ and $u_{\max }$ in $\alpha$ (for the optimal Youla parameter). For our example, both curves are depicted in Fig. 3 (top). Which constrained control problems are feasible, i.e. what values for $u_{\max }^{\text {des }}$ lead to a feasible control task? Proposition 3 gave a criterion for feasibility, that can be easily checked in Fig. 3: the constraint control problem is feasible, if and only if $u_{\max }^{\text {des }} \geq 7.5105$. Additionally, Fig. 3 (bottom) shows the tradeoff-curve over $\alpha$ in the $u_{\max } / e_{\max }$ plane and those combinations of maximum error and maximum control amplitude, that cannot be reached by any linear controller (in the considered parameterisation. This is comparable to the more "classical" tradeoff curves for RMS regulation error and RMS control signal within LQG control,
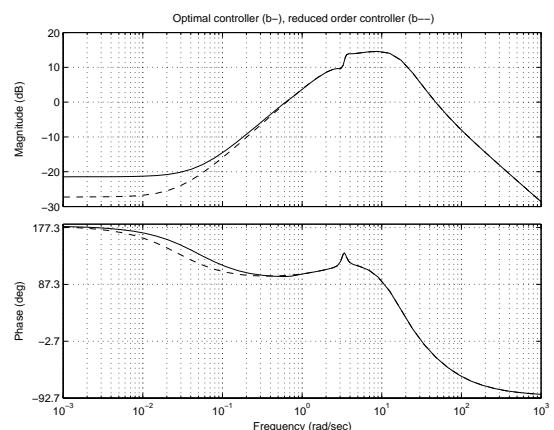

Figure 4: Optimal controller of order 8 (solid) and reduced order controller of order 5 (dashed).

cf. [3, sec.X.B.]. Such curves might also be helpful in an early design stage: suppose, the desired maximum control amplitude is a design parameters, for exampel when choosing the actuator. Then, a look at Fig. 3 helps to assess how much performance (in terms of maximum error) can be gained, when increasing $u_{\max }^{\text {des }}$. If for instance the error does not decrease significantly when increasing the control effort, then a "smaller" (and probably cheaper) actuator might be chosen as well.

Now, we are going to examine the designed control system. We pick $Q^{*}(\alpha)$ for $\alpha=0.6$ and calculate the optimal $8 t h$ order controller. According to Fig. 3, the control loop has a maximum error amplitude $e_{\max }=8.5972$ and a maximum control amplitude $u_{\max }=10.0475$ for all admissible reference signals.

Apart from the design of an optimal controller, Theorem 1 allows the a-posteriori analysis of an already existing control system. Consider for example a reduced version of the optimal controller, namely $\hat{K}_{\text {opt }}(s)=$ $-37.04 \frac{(s-38.13)(s-0.050)\left(s^{2}+0.59 s+10.83\right)}{(s-3.21)\left(s^{2}+0.65 s+11.63\right)\left(s^{2}+21.67 s+250.1\right)}$, which was obtained by square-root balanced truncation (of the stable part of the controller). Applying this reduced order controller to the plant, we obtain a maximum error amplitude $\hat{e}_{\max }=8.5973$ and a maximum control amplitude $\hat{u}_{\max }=10.0494$, i.e. about the same properties with a lower order controller. Both controllers are shown in Fig. 4.

\section{Conclusions}

We presented a method for designing constraint control systems which are optimal in the sense, that the smallest possible error between reference signal and plant output is achieved. The bound on the control signal is supposed to be hard. To avoid a conservative design, we described the set of admissible external signals (i.e. reference signals) by hard bounds on amplitude and rate, which appears in many practical situations. The underlying constrained and infinite dimensional optimization problem was reformulated to an unconstrained one, and approximated by a finite dimensional one, which is well suited for numerical solution, via non-linear optimisation, involving the solution of two linear programmes at each step. Finding 
a suitable starting point for the non-linear optimisation turned out not to be a problem. Using Youla parameterisation, this approach will typically end up with a high order controller. The presented technique, however, also allows the a-posterior check of a given controller. Hence, calculating a high order controller and then performing controller reduction along with an a-posteriori check of all specifications is an attractive step in this approach. The presented framework provides also a criterion to determine, if the constraint control problem is feasible for a given bound on the control signal.

\section{Acknowledgment}

This work was partly supported by the German research council DFG, while the author was with Paderborn University. Valuable discussions with N. Dourdoumas, D. Holtgrewe and V. Verdult are gratefully acknowledged.

\section{References}

[1] C. Barbu, R. Reginatto, A. R. Teel, and L. Zaccarian. Anti-windup for exponentially unstable linear systems with inputs limited in magnitude and rate. In Proc. of the American Control Conference, pages 12301234, Chicago, IL, USA, 2000.

[2] D. S. Bernstein and A. N. Michel. A chronological bibliography on saturating actuators. Int. J. of Robust and Nonlinear Control, 5:375-380, 1995. Special Issue Saturating Actuators.

[3] S. Boyd, C. Barrat, and S. Norman. Linear controller design: Limits of performance via convex optimization. Proc. of the IEEE, 78(3):529-574, Mar. 1990.

[4] N. O. DaCunha and E. Polak. Constrained minimization under vector valued criteria in infinite dimensional spaces. J. Math. Anal. Appl, 19:103124, 1967.

[5] J. A. D. Dona, R. Moheimani, and G. C. Goodwin. Robust combined PLC/LHG controller with allowed over-saturation of the input signal. In Proc. of the American Control Conference, pages 750-754, Chicago, IL, USA, 2000.

[6] E. G. Gilbert and K. T. Tan. Linear systems with state and control constraints: The theory and applications of maximal output admissible sets. IEEE Trans. on Automatic Control, 36(9):1008-1020, Sept. 1991.

[7] M. Green and D. J. N. Limebeer. Linear Robust Control. Prentice Hall, Englewood Cliffs, NJ, USA, 1995.

[8] D. Henrion, S. Tabouriech, and V. Kučera. Control of linear systems subject to input constraints: a polynomial approach. MIMO case. In Proc. of the American Control Conference, pages 1774-1778, Chicago, IL, USA, 2000.

[9] D. Holtgrewe. Entwurf von Mehrfachsystemen mit beschränkten Systemgrössen. PhD thesis, Dept of EE, University of Paderborn, 33095 Paderborn, Germany, 1992.

[10] T. Hu and Z. Lin. Control systems with actuator saturation: Analysis and design. Birkhäuser, Boston, MA, USA, July 2001.

[11] M. V. Kothare, V. Balakrishnan, and M. Morari. Robust Constrained Model Predictive Control using Linear Matrix Inequalities. Automatica, 32(10):1361-1379, Oct. 1996.

[12] M. V. Kothare, P. J. Campo, M. Morari, and C. N. Nett. A unified framework for the study of Antiwindup designs. Automatica, 30(12):18691883, Dec. 1994.

[13] A. Linnemann. Convergent Ritz approximations of the set of stabilizing controllers. Systems \& Control Letters, 36(2):151-156, Feb. 1999.

[14] R. W. Reichel. Entwurf von Regelsystemen mit Begrenzungen. Automatisierungstechnik, 34(11):446-453, Nov. 1986.

[15] W. Reinelt. Maximum Output Amplitude of Linear Systems for certain Input Constraints. In Proc. of the IEEE Conference on Decision and Control, pages 1075-1080, Sydney, Australia, Dec. 2000.
[16] W. Reinelt. Control of Multivariable Systems with Hard Constraints. In Proc. of the European Control Conference, Porto, Portugal, Sept. 2001.

[17] W. Reinelt and M. Canale. Robust control of SISO systems subject to hard input constraints. In Proc. of the European Control Conference, Porto, Portugal, Sept. 2001.

[18] A. Saberi, J. Han, and A. A. Stoorvogel. Constrained stabilization problems for linear plants. In Proc. of the American Control Conference, pages 4393-4397, Chicago, IL, USA, 2000.

[19] A. Saberi, A. A. Stoorvogel, and P. Sannuti. Control of Linear Systems with Regulation and Input Constraints. Communications and Control Engineering. Springer Verlag, London, UK, 2000.

[20] E. D. Sontag. An algebraic approach to bounded controllability of linear systems. Int. J. of Control, 39(1):181-188, Jan. 1984.

[21] A. A. Stoorvogel and A. Saberi. Special issue on control problems with constraints. Int. J. of Robust and Nonlinear Control, 9(10), 1999.

[22] H. J. Sussmann, E. D. Sontag, and Y. Yang. A general result on the stabilization of linear systems using bounded controls. IEEE Trans. on Automatic Control, 39(12):2411-2425, Dec. 1994.

[23] S. Tabouriech and G. Garcia, editors. Control of Uncertain Systems with Bounded Inputs, volume 227 of Lecture Notes in Control and Information Science. Springer Verlag, London, UK, 1997.

[24] T. Ting and K. Poolla. Upper bounds and approximate solutions for multidisk problems. IEEE Trans. on Automatic Control, 33(8):783-786, Aug. 1988.

[25] M. Vidyasagar. Control System Synthesis - A Factorization Approach. MIT Press, Cambridge, MA, USA, 1985. 